\section{Smoking habits and teachable moment among hospitalized patients admitted to the internal medicine department}

\section{Dennis Bösch}

Department of Internal Medicine/ Section of Pulmonology, Klinikum Bremerhaven, Germany

\section{Abstract}

Hospitalization seems a favorable setting to quit smoking. The purpose of this study was to determine the smoking habits and teachable moments among hospitalized patients admitted to the internal medicine department. A prospective study involving 253 patients admitted to an academic teaching hospital was conducted.

The rate of current smokers ranged from $75 \%$ in the group of 30 to 39 -year-old male patients to $0 \%$ in female patients 80 years and older; mean prevalence $34 \%$. Although males had a higher prevalence of smoking in the younger population, the number of current smokers between the genders nearly converged in patients greater or equal to 50 years of age. Among the internal subspecialties, it was highest in patients admitted for pulmonary disease. The number of patients that quit smoking before discharge was similar between both genders, but significantly different among the age-groups and subspecialties. The fraction of quitters was greater in patients 50 years and older, compared to patients less than 50 years (35\% vs. $11 \%$, respectively), and in patients admitted for pulmonary disease, compared to all other patients (33\% vs. $12 \%$, respectively). In summary, smoking habits among inpatients are very heterogenous, depending on age, gender, and medical reason for admission. Highest prevalences are found in young, male patients, as well as patients admitted for pulmonary disease. On the other hand, the rate of quitters is significantly greater in patients 50 years and older, and patients admitted for pulmonary disease. Besides targeting our efforts at those who are susceptible to the effects, within the scope of secondary and tertiary prevention of many chronic pulmonary and cardiac diseases, it might be favorable and most efficient to focus on the young, middle-aged patients in tailoring specific support, especially in respiratory medicine.

\section{Introduction}

Smoking is clearly associated with an increased risk of hospital admission and duration of hospitalization. ${ }^{1}$ Consequently, we find a fair amount of currently smoking patients in hospitals worldwide. On the other hand, hospitalization seems a favorable setting to quit smoking. For various reasons, initiating and supporting smoking cessation attempts of hospitalized patients is a promising step to contribute to a reduction of smoking and smokingrelated morbidity. Not only are worldwide extending rules and legal regulations making it effectively difficult to smoke while being hospitalized, the illness itself often restricts the ability and urge to smoke during hospitalization. Furthermore, in many cases we have the chance to reflect on the connection between smoking behavior and disease and make hospitalization a teachable moment. ${ }^{2,3}$ The term teachable moment in general has been used to describe naturally occurring life transitions or health events thought to motivate individuals to spontaneously adopt riskreducing health behaviors. ${ }^{2}$ Also, it has been shown that confrontational counseling increases risk perceptions and self-efficacy, and decreases self-exempting beliefs (risk denial) in smokers, making hospitalization a valuable instrument. ${ }^{4}$

Currently, approximately half of US hospitals have adopted a smoke-free hospital campus policy. ${ }^{5}$ However, despite existing regulations, a significant number of smokers admit to smoking during their hospital stay, depending on nicotine withdrawal symptoms. ${ }^{6}$ Various programs for a hospital-based smoking cessation service have been promoted worldwide. ${ }^{7-9}$ Still, studies of different interventional measures to support smoking cessation among hospitalized patients have shown inconsistent and overall rather improvable results. ${ }^{9-14}$ One reason might be the heterogeneity of the patients admitted to the hospital. These patients include women and men of all ages, with or without smoking related disease, different smoking habits and extent of addiction. Also, the prevalence among the hospitalized patients, and with this the awareness of the problem, seems to vary tremendously between the different specialties. Overall, prevalence rates of currently smoking inpatients vary between $17 \%$ and $63 \%$ in general and psychiatric departments, respectively. ${ }^{15,16}$ Published data about differentiated characteristics of hospitalized patients in regard to smoking behavior and relation to age, gender, underlying disease etc. is very sparse. On the other hand, it seems reasonable to take a closer look at our patients before we can try to improve our interventional tools (smoking cessation counseling etc.). Further need of hospitalbased research, addressing specific cessation strategies, has been previously proclaimed and demanded. ${ }^{17}$ About $7.5 \%$ of hospital admissions are attributed to smoking. ${ }^{18}$ This holds espe-
Correspondence: Dennis Bösch, Klinikum Bremerhaven, Reinkenheide Medizinische Klinik 1, Sektion Pneumologie, Postbrookstr. 103, 27574, Bremerhaven, Germany.

E-mail: _ dennis.boesch@klinikumbremerhaven.de

Key words: smoking; smoking habits, hospitalization, smoking cessation, smoking cessation support, teachable moment.

Conflicts of interests: the authors declare that they have no conflicts of interests.

Received for publication: 20 December 2011

Revision received: 7 March 2012.

Accepted for publication: 8 March 2012.

This work is licensed under a Creative Commons Attribution NonCommercial 3.0 License (CC BYNC 3.0).

CC Copyright D. Bösch, 2012

Licensee PAGEPress, Italy

Chest Disease Reports 2012; 2:e6

doi:10.4081/cdr.2012.e6

cially true for internal medicine and makes this department favorable for supporting smoking cessation.

This survey was designed to obtain further insight about the smoking behavior, as well as the teachable moments in different age-groups and gender among hospitalized patients admitted for internal medicine related diseases.

\section{Materials and Methods}

Over a period of 10 weeks, data was collected and analyzed from 253 consecutive patients who were admitted to a ward in the internal medicine department of a 730-bed academic teaching hospital. This study was approved by the institutional review board of the local medical association.Even though, there were no exclusion criteria, due to lack of reliability of the available data, 3 patients with coexisting mental illness could not be taken into consideration and were exclude prior to data acquisition. All data was otherwise gathered from the admission history sheet and personal contact with the patients and then anonymously administered. Current smokers were defined as individuals who smoked regularly (at least one cigarette per day). Patients who had quit smoking, at least one week prior admission, were considered former smokers, and those who had never smoked regularly or irregularly were considered never smokers.

All statistical analyses were conducted using Numbers ‘09, Apple Inc., California, USA, and 
Prism 5, GraphPad Software Inc., California, USA. After descriptive statistics, data was plotted in age-groups and adjusted for gender and reason for admission. For the comparison between the age-groups and the underlying reason for hospitalization, the chi-square test has been used. A P value less than 0.05 was applied to determine statistical significance.

\section{Results}

The survey encompassed the data of 253 patients. The study group was composed of 148 males and 105 females, $58.5 \%$ and $41.5 \%$, respectively. The mean age was 64.8 years (ranging from 18 to 95 years). The majority of patients (142 patients, 56\%) were admitted for pulmonary disease. Of the total 253 patients, 86 (34\%) were current smokers, 101 (40\%) were former smokers, and 66 (26\%) were never smokers at the time of admission. The patients' demographics and smoking habits among the subspecialties are summarized in Table 1.

The smoking habits between the genders and along the age groups were very different. The prevalence of current smokers ranged from $75 \%$ in the group of 30 to 39 -year-old male patients to $0 \%$ in female patients 80 years and older. The distribution of smokers, ex-smokers, and never-smokers among all age groups is shown in Figures 1 and 2 for males and females, respectively. Comparing both genders, the prevalence of current smokers up to 40 yearolds was higher in male patients. It exceeded the prevalence rates in young female patients by more than $20 \%$. Most of the current smokers among the female patients were found in those 40 to 49 years-old compared to the male patients who were only 30 to 39 year old. In male and female patients 40 years and older, the courses of prevalences along the age groups ran rather concurrent, while male patients had a slightly higher prevalence level.

Interestingly, the fraction of never-smokers was distinctly higher in female compared to male patients, being above $30 \%$ and below $20 \%$, respectively. Contrary to the rates of current-smokers, the prevalence rates of ex-smokers increased with age in both genders. The crossover point when the rate of ex-smokers exceeds the rate of smokers was within the group of 60 to 69 year-old in both, male and female patients.

Comparing the average number of cigarettes smoked daily, young female smokers (under 40 years of age) smoked less than young male smokers, 12.0 vs. 15.4 cigarettes per day, respectively. Overall there was no significant difference inthe average number of cigarettes smoked daily between males and females (male 18.3 and females 16.9 cigarettes per day).
While the prevalence of current-smokers at the time of admission was $34 \%$ among all patients, it decreased to $25 \%$ at the time of discharge, according a mean quitting rate of $27 \%$. The rate of patients that stopped smoking while hospitalized was quite similar between both genders, but different among the agegroups. The highest quitting rate was $50 \%$ and was found in patients being 50 to 59 years of age. The rate of current-smokers at admission and discharge among all age-groups is shown in Figure 3, Comparing the patients up to 50 years and 50 years and older, it becomes apparent that the rate of patients who quit smoking was significantly higher in the group 50 years and older, being $35 \%$, compared to $11 \%$ in patients younger than 50 years $(\mathrm{P}=0.019)$.

Between the subspecialties, the highest rates of smokers and ex-smokers were found in patients with underlying pulmonary disease, $42 \%$ and $41 \%$, compared to rates of $23 \%$ and $39 \%$ in all other patients (Table 1). The comparison of patients who quit smoking until discharge among the different subspecialties is shown in Table 2. The fraction of patients who stopped smoking before discharge was significantly larger in patients admitted for pulmonary disease in relation to all other patients, $33 \%$ vs. $12 \%$, respectively $(\mathrm{P}=0.036)$ (Figure 4).

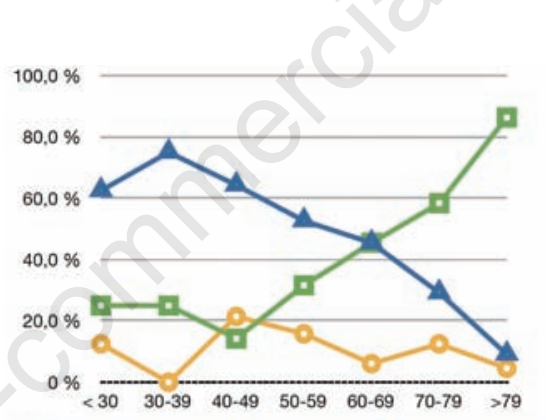

$\triangle$ Current-smokers $\square$ Ex-smokers 0 Never-smokers

Figure 1. Smoking habits among male patients.

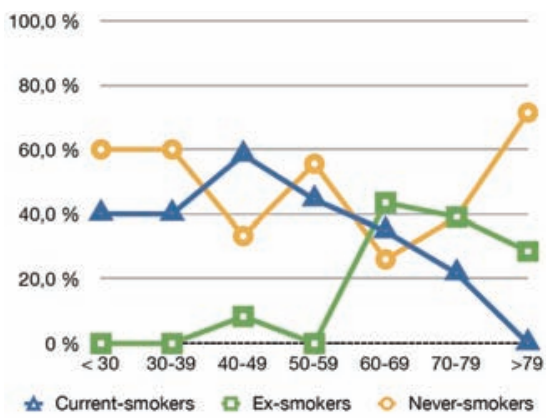

Figure 2. Smoking habits among female patients.

\section{Discussion}

This study has several interesting findings. First, the prevalence of current-smokers among the patients was very heterogeneous, depending on age, gender, and medical reason for admission. While the mean rate of currentsmokers was $34 \%$, highest prevalence was found in male patients up to 50 years of age and patients admitted for pulmonary disease; $65 \%$, and $42 \%$, respectively.

Secondly, the rate of patients that (spontaneously) quit smoking before discharge was significantly greater in older patients (50 years and older) and patients admitted for pulmonary disease; $35 \%$, and $33 \%$, respectively; compared to the overall rate of $27 \%$.

It is well known that smoking leads to morbidity and hospitalization, and that a fair amount of hospitalized patients are current smokers or former smokers. We also know that high intensity behavioral interventions that begin during a hospital stay and include at least one month of supportive contact after discharge promote smoking cessation among hospitalized patients, and that these interventions are effective regardless of the patient's admitting diagnosis. ${ }^{11}$ Interventions of lower

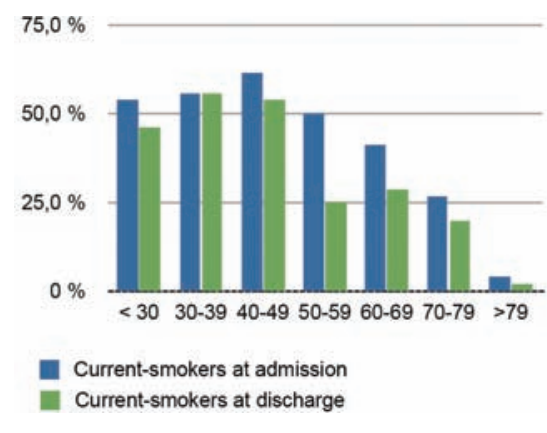

Figure 3. Prevalence of current-smokers at admission and at discharge among all agegroups. Difference between patients younger than 50 and 50 and older $(\mathbf{P}=\mathbf{0 . 0 1 9})$.

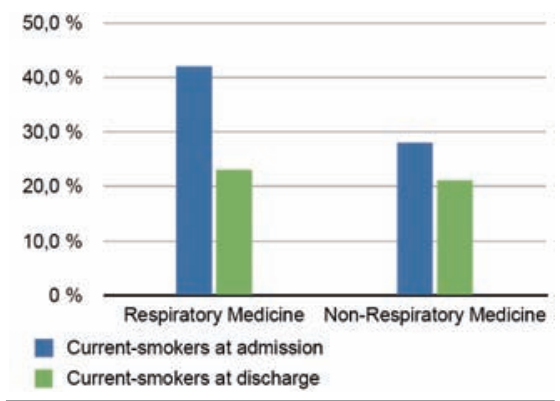

Figure 4. Prevalence of current-smokers at admission and at discharge for patients admitted for underlying pulmonary disease and all other patients. Difference between quitters among patients with pulmonary disease and others $(P=0.036)$. 
Table 1. Patients demographics and smoking habits among the subspecialties.

\begin{tabular}{lcccccc} 
Characteristic & Total & Respiratory medicine & Cardiology & Gastroenterology & Nephrology & Miscellaneous \\
Patients, No (\%) & $253(100)$ & $142(56)$ & $23(9)$ & $49(19)$ & $16(6)$ & $23(9)$ \\
Male, No (\%) & $148(58.5)$ & $91(64)$ & $13(57)$ & $27(55)$ & $5(31)$ & $12(52)$ \\
\hline Female, No (\%) & $105(41.5)$ & $51(36)$ & $10(43)$ & $22(45)$ & $11(69)$ & $11(48)$ \\
Age & $64.8(18-95)$ & $62.1(18-88)$ & $68.7(38-90)$ & $65.1(18-95)$ & $77.1(65-88)$ & $68,3(26-91)$ \\
\hline Current smokers, No (\%) & $86(34)$ & $60(42)$ & $8(35)$ & $13(27)$ & $4(25)$ & $1(4)$ \\
Former smokers, No (\%) & $101(40)$ & $58(41)$ & $8(35)$ & $15(31)$ & $6(38)$ & $14(61)$ \\
\hline Never smokers, No (\%) & $66(26)$ & $24(17)$ & $7(30)$ & $21(43)$ & $6(38)$ & $8(35)$ \\
Cigarettes smoked per day (SD) & $17.9(6.9)$ & $18.3(6.0)$ & $14.4(6.3)$ & $18.4(10.8)$ & $17.5(4.3)$ & $18(0)$ \\
\hline
\end{tabular}

Table 2. Smoking cessation before discharge among the subspecialties.

\begin{tabular}{lcccccc} 
Characteristic & Total & Respiratory medicine & Cardiology & Gastroenterology & Nephrology & Miscellaneous \\
Current smokers, No (\%) & $86(100)$ & $60(70)$ & $8(9)$ & $13(15)$ & $4(5)$ & $1(1)$ \\
Smoking cessation at discharge, No (\%) & $23(27)$ & $20(33)$ & 0 & $1(8)$ & $2(50)$ & 0 \\
\hline
\end{tabular}

intensity or shorter duration have not been shown to be as effective in this setting, ${ }^{11}$ and there is room for quality improvement in many programs ${ }^{19}$ Besides suboptimal utilization of medication (nicotine replacement therapy), there are several reasons that hamper the efficacy of attempts to quit smoking within the hospital settings such as cutbacks on the length of hospital stay, different needs between heterogeneous patient population, inconsistent education of physicians, nurses etc. Looking at the heterogeneous patient population, it seems obvious that you need a different approach and assisting counseling material e.g. a 20 -year-old male patient or a 70year-old female patient. A first step in tailoring adjusted measures should be profiling our patients to be able to effectively meet their demands. Unfortunately, published data about differentiated characteristics of patients hospitalized in the internal medicine department are very sparse. A smaller study has shown a rate of current smokers of $17 \%$ in patients admitted to a general hospital (in Brazil), and demonstrated that smokers were rather young, male, and admitted into specific departments: psychiatry, pulmonology, ENT, cardiovascular surgery, and emergency medicine. ${ }^{15}$ Another study illustrated a rate of $37 \%$ among all hospital admission (of a regional hospital in Canada), and pointed out that $51 \%$ were admitted with smoking-related disease. ${ }^{20}$ Among psychiatric patients in a Swiss hospital, a rate of $63 \%$ was found. ${ }^{16}$ To the best of my knowledge, the current study is the first that demonstrates differentiated data of the smoking habits of patients admitted to an internal medicine department, as well as their tendency for quit attempts without special intervention.

Of the total 253 patients screened, $34 \%$ were considered current smokers. Less surprisingly, the highest rates of current smokers were found in pulmonary and cardiologic patients, $42 \%$, and $35 \%$, respectively. However, the mean prevalence rate alone is not helpful in the attempt to adapt specific offers in smoking cessation support in hospitalized patients with an age span from 18 to 95 years. A closer look revealed that the rate of current-smokers was extremely variable between the genders and age groups. The biggest challenge are probably young male smokers, showing a prevalence of $67 \%$ (male patients up to 39 years of age) and on the other hand a low spontaneous drive to quit smoking; spontaneous quitting-rate $12.5 \%$. With the omitted special effort in smoking cessation support at the study time, the effect can be assessed as teachable moment. While rather immobile patients often undergo 'involuntary quitting' as a result of the smokefree legislation and hospital policy, patients that are mobile or gain mobility within the course of reconvalescence have the chance to visit an outdoor smoking area.

While males had a higher prevalence level of current-smokers in younger patients, the levels between the genders converged in the patients 50 and older. While it is known that females are more likely to be motivated to quit by life changes, and are more likely to use medications or counseling for smoking cessation than males, ${ }^{21}$ current results didn't show a significant difference in spontaneous quitters between males and females, $27.6 \%$, and $25 \%$, respectively.

Overall, we found more current-smokers among the patients under 60 years of age. At the same time, this group of young and middleaged patients accounts for only $30 \%$ of all patients admitted. So, focusing on the young and middle-aged patients seems economically interesting. While the middle-aged patients (50 to 59 years) seem to have the highest drive to quit smoking, the patients up to 49 years of age have the lowest, and probably need more intense attention. On the other hand, initial hospitalization has previously been found to be an independent predictor of smoking abstinence after discharge from hospital in patients who had received smoking cessation intervention, ${ }^{22}$ and as a natural fact, initial hospitalization is more likely in younger patients.

Smoking cessation is known to be a very important measure within the scope of secondary and tertiary prevention in many chronic pulmonary and cardiac diseases, such as chronic obstructive pulmonary disease, etc. So, besides targeting those who are especially suitable for intensive measures of smoking cessation support, we have to focus on those who obviously and directly benefit from smoking cessation.

Compared to a German health survey, ${ }^{23}$ the data of the region concerned lay within the prevalence rate of our hospitalized population; overall 32.1 vs. $34.0 \%$, males 38.0 vs. $39.2 \%$, and females $26.4 v$ s. $26.7 \%$. The higher prevalence rate upon the studied region compared to the general rate in Germany, 32.1 vs. $27.6 \%$ is mostly explained by a lower local socioeconomic status. Still, the regional prevalence of $32 \%$ lies well within the average of most western countries, ${ }^{24}$ so it may be presumed that the presented data is representative to many other countries as well.

The presented study has several limitations. Even though the number of patients seems to be representative for those admitted for pulmonary disease, it appears to be sparse for all other indications. Furthermore, the collected data could have been more detailed and the interrogation more standardized. It also needs to be stressed that the spontaneous quitting rate before discharge does not necessarily mean long term cessation and the present study is lacking this information. 
In conclusion, the smoking habits among patients admitted to the internal medicine department are very heterogeneous. Depending on age, gender, and medical reason for admission, we found variable rates of current-smokers. The highest prevalences prevail in the male patients up to 50 years of age, as well as the patients admitted for pulmonary disease. On the other hand, the rate of patients that quit smoking before discharge are significantly greater in patients 50 years and older, as well as patients admitted for pulmonary disease. Although supporting smoking cessation in hospitalized patients is a necessity independently of age, gender, or underlying disease, taking the available data under consideration, it might be favorable and most efficient to focus on the young and middle-aged patients in tailoring specific support, especially in respiratory medicine, besides those who are particularly susceptible to the effects, within the scope of secondary and tertiary prevention of many chronic pulmonary and cardiac diseases.

\section{References}

1. Hvidtfeldt UA, Rasmussen S, Grønbæk M, et al. Influence of smoking and alcohol consumption on admissions and duration of hospitalization. Eur J Public Health 2009 Sep 30. [Epub ahead of print]

2. McBride CM, Emmons KM, Lipkus IM. Understanding the potential of teachable moments: the case of smoking cessation. Health Educ Res 2003;18:156-70.

3. Gritz ER, Fingeret MC, Vidrine DJ, et al. Successes and failures of the teachable moment - smoking cessation in cancer patients. Cancer 2006;106:17-27.

4. Kotz D, Huibers MJ, West RJ, et al. What mediates the effect of confrontational counselling on smoking cessation in smokers with COPD? Patient Educ Couns 2009;76:16-24.
5. Williams SC, Hafner JM, Morton DJ, et al. The adoption of smoke-free hospital campuses in the United States. Tob Control 2009;18:451-8.

6. Rigotti NA, Arnsten JH, McKool KM, et al. Smoking by patients in a smoke-free hospital: prevalence, predictors, and implications. Prev Med 2000;31:159-66.

7. Bolliger CT, van Biljon X, Humair JP, et al. Promoting hospital-based smoking cessation services at major Swiss hospitals: a before and after study. Swiss Med Wkly 2008;138:427-31.

8. Freund M, Campbell E, Paul C, et al. Smoking care provision in hospitals: a review of prevalence. Nicotine Tob Res 2008;10:757-74.

9. Borglykke A, Pisinger C, Jørgensen T, Ibsen $H$. The effectiveness of smoking cessation groups offered to hospitalised patients with symptoms of exacerbations of chronic obstructive pulmonary disease (COPD). Clin Respir J 2008;2:158-65.

10. Bösch D. [Smoking-cessation-counselling as a smoking cessation interventional tool for hospitalized patients - results of a pilot study.] Pneumologie 2009;63:578-81. [Article in German].

11. Rigotti NA, Munafo MR, Stead LF. Interventions for smoking cessation in hospitalised patients. Cochrane Database Syst Rev 2007;(3):CD001837.

12. Freund M, Campbell E, Paul C, et al. Increasing smoking cessation care provision in hospitals: a meta-analysis of intervention effect. Nicotine Tob Res 2009;11: 650-62.

13. Reid RD, Mullen KA, Slovinec D'Angelo ME, et al. Smoking cessation for hospitalized smokers: an evaluation of the "Ottawa Model". Nicotine Tob Res 2010;12:11-8.

14. Hjalmarson A, Boëthius G. The effectiveness of brief advice and extended smoking cessation counseling programs when implemented routinely in hospitals. Prev Med 2007;45:202-7.
15. de Oliveira MVC, de Oliveira TR, de Castro Pereira CA, et al. Smoking among hospitalized patients in a general hospital. J Bras Pneumol 2008;34: 936-41.

16. Keizer I, Gex-Fabry M, Eytan A, Bertschy G. Smoking in psychiatric inpatients: association with working status, diagnosis, comorbid substance abuse and history of suicide attempts. Addict Behav 2009;34: 815-20.

17. Wolfenden L, Campbell E, Wiggers J, et al. Helping hospital patients quit: What the evidence supports and what guidelines recommend. Prev Med 2008;46:346-57.

18. Cruts AA, Feenstra TL, van Laar MW. Morbidity due to smoking in the Netherlands: an estimated 90,000 clinical hospital admissions in 2005. Ned Tijdschr Geneeskd 2008;152:1469-72.

19. Faseru B, Turner M, Ruder C, et al. Evaluation of a hospital-based tobacco treatment servive: outcomes and lessons learned. J Hosp Med 2011;6:143-50.

20. Senior SL. Study of smoking habits in hospital and attitudes of medical staff towards smoking. Can Med Assoc J 1982;126:131-3.

21. Reid RD, Pipe AL, Riley DL, Sorensen M. Sex differences in attitudes and experiences concerning smoking and cessation: results from an international survey. Patient Educ Couns 2009;76:99-105.

22. Ong KC, Cheong GN, Prabhakaran L, Earnest A. Predictors of success in smoking cessation among hospitalized patients. Respirology 2005;10:63-9.

23. Statistisches Bundesamt. Mikrozensus 2009 - Rauchgewohnheiten der Bevölkerung [Microcensus 2009, smoking habits of the population]. Wiesbaden: Statistisches Bundesamt; 2010.

24. WHO report on the global tobacco epidemic, 2009: Implementing smoke-free environments. World Health Organization. Geneva; 2009. 